\title{
Sodium selenite inhibits leukemia HL-60 cell proliferation and induces cell apoptosis by enhancing the phosphorylation of JNK1 and increasing the expression of p21 and p27
}

\author{
SUN WU ${ }^{1 *}$, YONGHUA BAO $^{2 *}$, DONG MA $^{1}$, YOUMEI ZI ${ }^{1}$, CUI YANG $^{1}$, MAN YANG $^{1}$, \\ MENGTAO XING ${ }^{4}$ and WANCAI YANG ${ }^{3,4}$
}

\begin{abstract}
${ }^{1}$ Department of Hematology, The First Affiliated Hospital of Xinxiang Medical University, Weihui, Henan; Departments of ${ }^{2}$ Immunology and ${ }^{3}$ Pathology, Xinxiang Medical University, Xinxiang, Henan, P.R. China;

${ }^{4}$ Department of Pathology, University of Illinois at Chicago, Chicago, IL, USA
\end{abstract}

Received January 28, 2014; Accepted June 30, 2014

DOI: $10.3892 /$ ijmm.2014.1854

\begin{abstract}
Selenium is an essential trace element and has shown chemopreventive or therapeutic activities on human solid cancers; however, whether it has anticancer effects on leukemia has not yet been elucidated. The present study was designed to determine the role of selenium on HL-60 human promyelocytic leukemia cells. We found that $100 \mathrm{nM}$ of sodium selenite (Se) had no significant effects on cell proliferation, apoptosis and the cell cycle; however, a higher concentration of $250 \mathrm{nM}$ of Se significantly inhibited cell proliferation, promoted apoptosis and induced cell cycle arrest at the $\mathrm{S}$ phase after $48 \mathrm{~h}$ of treatment $(\mathrm{P}<0.05)$, thus demonstrating the anticancer activities of selenium in leukemia. However, the decrease in c-Jun NH2-terminal kinase 1 (JNK1) expression by targeting JNK1 using small interfering RNA attenuated the inhibitory effects of Se on cell proliferation and the induction of apoptosis. Mechanistic studies showed that the anticancer activities of Se were associated with the enhanced phosphorylation of JNK1 and the increased expression of the cell cycle regulators, p21 and p27, as well as the downregulation of cyclin D1. Our data provide further evidence that the appropriate concentration of selenium has therapeutic potential in leukemia.
\end{abstract}

Correspondence to: Dr Yonghua Bao, Department of Immunology, Xinxiang Medical University, 601 East Jinsui Ave, Xinxiang, Henan 453003, P.R. China

E-mail: baoyonghua2005@126.com

Professor Wancai Yang, Department of Pathology, Xinxiang Medical University, 601 East Jinsui Ave, Xinxiang, Henan 453003, P.R. China E-mail: wyang06@uic.edu

*Contributed equally

Abbreviations: Se, selenium; JNK1, c-Jun NH2 terminal kinase 1

Key words: selenium, leukemia, proliferation, apoptosis, c-Jun NH2terminal kinase $1, \mathrm{p} 21, \mathrm{p} 27$

\section{Introduction}

Leukemia is one of the most comment malignant diseases worldwide. Epidemiological studies have shown that in the United States, among males aged $>40$ years, leukemia is the most common fatal cancer; among females, leukemia is the leading cause of cancer-related mortality before the age of 20 . In children (newborns to 14 years of age) almost one third of cancer cases are diagnosed are leukemia (particularly acute lymphocytic leukemia) (1). Chemotherapy remains the major strategy for the treatment of leukemia; however, traditional therapy has lower efficacy and severe side-effects. Therefore, it is urgent to develop an alternative medicine or alternative therapeutic approaches for the treatment of leukemia. It has been demonstrated that the trace element, selenium, has potential effects on leukemia (2).

Selenium plays important roles in different physiological functions of the human body. Epidemiological and clinical studies have reported that the inadequate status of selenium increases the risk of cancer (3). Basic research and clinical trials have demonstrated the protective effects of selenium against prostate, colorectal and other solid cancers (4-9). The anticancer effects of selenium have been postulated to be associated with the inhibition of cell proliferation and the induction of apoptosis through different signaling pathways, particularly its antioxidant and anti-inflammatory effects which are mediated through the activity of selenoenzymes (9). Selenium compounds have also been shown to be involved in the mitochondrial pathway, protein kinases, tumor necrosis factor, the activation of caspases and reactive oxygen species $(3,7,8)$.

We have previously demonstrated that the anticancer effects of selenium are mediated through the activation of c-Jun NH2-ternimal kinase 1 (JNK1) and the inhibition of the Wnt/ $\beta$-catenin signaling pathway in colorectal cancer in mouse models and colorectal cancer cell lines (10). JNK1 is a member of the mitogen-activated protein kinase (MAPK) family, and plays a critical role in the regulation of cell proliferation, differentiation and apoptosis (11-14). In previous studies, we demonstrated that JNK1 plays synergistic role with the cell cycle regulator, p21 (12), and that activated JNK1 
(JNK1 phosphorylation) interacts with and downregulates $\beta$-catenin signaling (15). In fact, $\mathrm{p} 21$ and $\mathrm{Wnt} / \beta$-catenin are negatively interacted through c-myc and cyclin D1 (16-18). Whether selenium also exerts anticancer effects by promoting apoptosis and inhibiting cell proliferation in leukemia cells, and the underlying mechanisms involved, remains unclear.

In the present study, using HL-60 human promyelocytic leukemia cells, we found that a higher concentration of selenium significantly inhibited cell proliferation, induced apoptosis, as well as changes in the cell cycle, which were associasted with the enhanced phosphorylation of JNK and the increased expression of $\mathrm{p} 21$ and p27, and with the deceased expression of cyclin D1.

\section{Materials and methods}

Cell lines and chemicals. HL-60 human promyelocytic leukemia cells obtained from the American Type Culture Collection (ATCC; Manassas, VA, USA) were maintained in RPMI-1640 medium (Life Technologies, Inc., Carlsbad, CA, USA) supplemented with $10 \%$ fetal bovine serum (FBS) and antibiotics (10,000 U/ml penicillin and $10 \mu \mathrm{g} / \mathrm{ml}$ streptomycin). The cells were cultured at $37^{\circ} \mathrm{C}$ in a humidified atmosphere containing $5 \% \mathrm{CO}_{2}$. Sodium selenite (Se) was purchased from Sigma-Aldrich (St. Louis, MO, USA).

Cell proliferation assay. A total of $1 \times 10^{4}$ HL-60 cells was seeded in each well of a 96-well plate and incubated overnight. The medium was replaced with fresh medium with a final concentration of Se (100 or $250 \mathrm{nM})$. Phosphate-buffered saline (PBS) (0 nM of Se) was used as a control. Three independent experiments were performed. Followign 24 and $48 \mathrm{~h}$ of exposure to $\mathrm{Se}$, cell proliferation was determined by 3-(4,5-dimethylthiazol-2-yl)-5-(3-carboxymethoxyphenyl)-2(4-sulfophenyl)-2H-tetrazolium (MTS) assay using the CellTiter 96 Non-Radioactive Cell Proliferation Assay kit according to the manufacturer's instructions (Promega Corp., Madison, WI, USA).

Apoptosis and cell cycle analysis. To detect apoptosis, the HL-60 cells were treated with various concentrations of $\mathrm{Se}$ $(0,100$ and $250 \mathrm{nM})$ After $48 \mathrm{~h}$ of treatment, the cells were harvested and fixed with $70 \%$ ethanol followed by propidium iodide (PI) staining. The cells were then counted using a flow cytometer (FACScan; BD Biosciences, San Jose, CA, USA) to detect apoptosis and for analysis of the cell cycle. Usually, approximately 10,000 cells were counted. The percentage of apoptotic cells was calculated by dividing the total number of cells by the number of apoptotic cells (e.g., the number of apoptotic cells/the total number of harvested cells). Cell cycle changes were assayed by dividing the total number of analyzed cells by the number of cells at individual cell cycle phases.

Effects of a decrease in JNK1 expression on cell proliferation and apoptosis. Small interfering RNA (siRNA) targeting human JNK1 (si-JNK1) (Sigma-Aldrich; sequence of si-JNK1 was 5'-GGGAUUUGUUAUCCAAAAU-3') was transfected into the HL-60 cells. Twenty-four hours after transfection, the cells were treated with $250 \mathrm{nM}$ of Se for $48 \mathrm{~h}$. siRNA for human green fluorescent protein (GFP; si-Control) (Sigma-Aldrich) was used as a

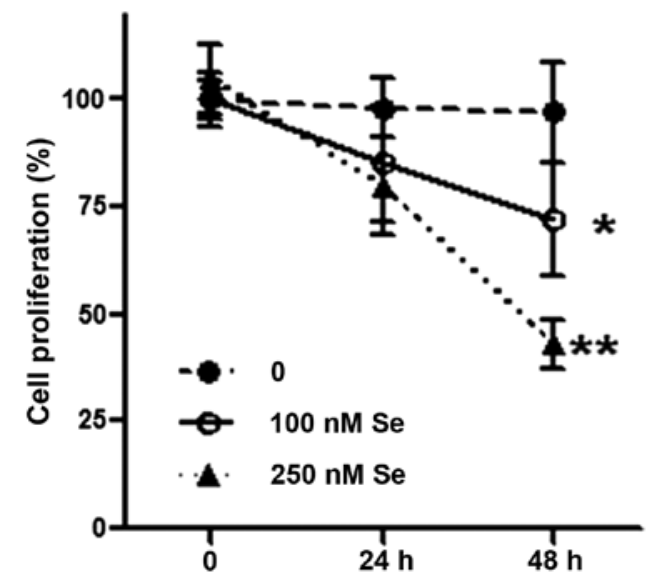

Figure 1. Sodium selenite (Se) inhibits the proliferation of HL-60 cells. The HL-60 cells were treated with 0,100 or $250 \mathrm{nM}$ of Se for 24 or $48 \mathrm{~h}$, and cell proliferation (percentage inhibition rate) was analyzed by MTS assay. ${ }^{*} \mathrm{P}<0.05$ and $^{* *} \mathrm{P}<0.01$, compared to the cells treated with 0 and $100 \mathrm{nM}$ Se, respectively.

control. Cell proliferation was determined by MTS assay, and apoptosis was analyzed by flow cytometry, as described above.

Immunoblotting. For immunoblotting, the cells were collected $48 \mathrm{~h}$ following treatment with Se. The cells were lysed using 1X RIPA buffer (Upstate Biotechnology, Lake Placid, NY, USA) containing a protease inhibitor cocktail (Sigma-Aldrich). Following cell lysis, $30 \mu \mathrm{g}$ of protein were loaded on a $10 \%$ SDS gel followed by transfer onto PVDF membranes. Antibodies against JNK1 and phosphorylated JNK1 (Cell Signaling Technology, Danvers, MA, USA), p21, p27 and cyclin D1 (Santa Cruz Biotechnology, Inc., Santa Cruz, CA, USA), and $\beta$-actin (1:10,000; Sigma-Aldrich) were used. The anti-mouse secondary antibody was purchased from Santa Cruz Biotechnology, Inc. The detected signals were visualized by an enhanced chemiluminescence reaction system, as recommended by the manufacturer (ECL-Plus; Amersham, Piscataway, NJ, USA). The immunoblotting intensities were quantified using Quantity One Software (Bio-Rad Laboratories, Inc., Hercules, CA, USA).

Statistical analysis. The significance of the differences between the means of the various subgroups was assessed by an unpaired two-tailed Student's t-test. Data are presented as the means $\pm \mathrm{SD}$. A value of $\mathrm{P}<0.05$ was considered to indicate a statistically significant difference.

\section{Results}

Se inhibits HL-60 cell proliferation. Se $(250 \mathrm{nM})$ significantly inhibited HL-60 cell proliferation after $48 \mathrm{~h}$ of treatment $(\mathrm{P}<0.01$, compared to the cells treated with 0 and $100 \mathrm{nM} \mathrm{Se})$, although $100 \mathrm{nM}$ of Se also exerted slight inhibitory effects on cell proliferation $(\mathrm{P}<0.05$, compared to the cells treated with 0 and $100 \mathrm{nM} \mathrm{Se}$ ) (Fig. 1).

Se promotes the apoptosis of HL-60 cells. The HL-60 cells were treated with 0,100 or $250 \mathrm{nM}$ of Se for $48 \mathrm{~h}$, and the cells were then collected for the analysis of apoptosis. Se $(100 \mathrm{nM})$ induced HL-60 cell apoptosis, although the induction was not 


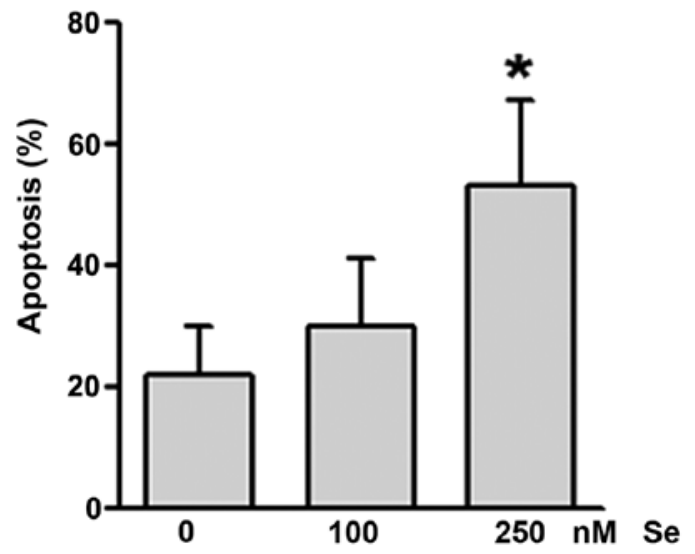

Figure 2. Sodium selenite (Se) induces the apoptosis of HL-60 cells. The HL-60 cells were treated with 0,100 or $250 \mathrm{nM}$ of Se for $48 \mathrm{~h}$ and collected for propidium iodide (PI) staining for the analysis of apoptosis, assayed by flow cytometry. Bars represent the mean (percentage of apoptosis of the total analyzed cells) \pm standard deviation $(\mathrm{SD}) .{ }^{*} \mathrm{P}<0.05$, compared to the cells treated with 0 and $100 \mathrm{nM}$ Se.

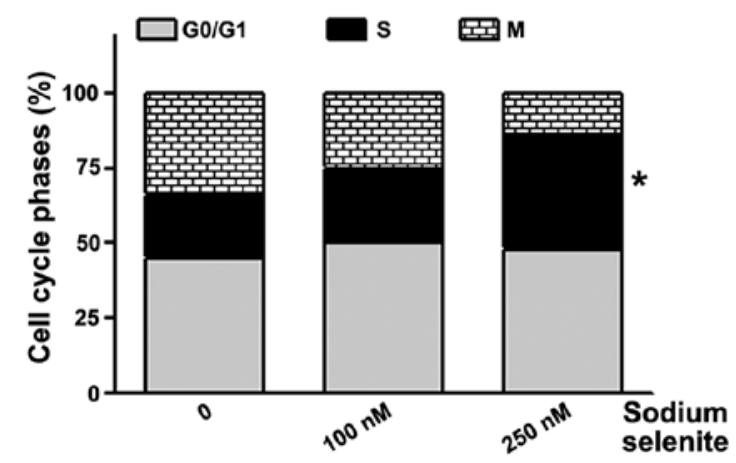

Figure 3. Sodium selenite induces cell cycle arrest at the S phase in HL-60 cells. The HL-60 cells were treated with 0,100 or $250 \mathrm{nM}$ of sodium selenite for $48 \mathrm{~h}$ and collected for propidium iodide (PI) staining for cell cycle analysis, assayed by flow cytometry. Bars represent the average of the percentage of cells in each individual phase of the total analyzed cells. ${ }^{*} \mathrm{P}<0.05$ compared to the cells treated with 0 and $100 \mathrm{nM}$ sodium selenite.

significant (Fig. 2). However, compared to the cells treated with 0 and $100 \mathrm{nM}$ Se, the higher concentration of Se $(250 \mathrm{nM})$ significantly induced apoptosis $(\mathrm{P}<0.05)$.

Se causes changes in the cell cycle in HL-60 cells. Following treatment with Se for $48 \mathrm{~h}, 100 \mathrm{nM}$ of Se caused changes in the cell cycle; however, these changes were not significant, although the percentage of cells in the M phase decreased and that of cells in the $\mathrm{S}$ phase increased compared to the untreated cells (Fig. 3). The higher concentration of Se $(250 \mathrm{nM})$ significantly caused changes in the cell cycle in HL-60 cells; in particular, Se induced cell cycle arrest at the $\mathrm{S}$ phase $(\mathrm{P}<0.05$, compared to the cells treated with 0 and $100 \mathrm{nM} \mathrm{Se}$ ).

Decrease in JNK1 expression attenuates the effects of Se on cell proliferation and apoptosis. In order to determine the importance of JNK1 in the inhibitory effects of Se on cell proliferation and its apoptosis-promoting effects, we transfected the HL-60 cells with siRNA targeting human JNK1, and subsequently treated the
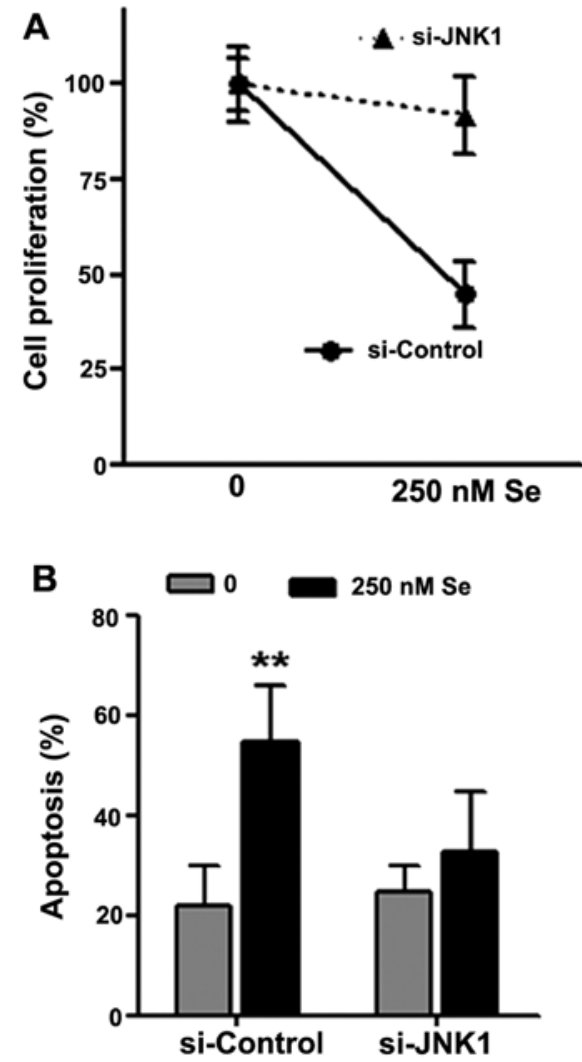

Figure 4. Decrease in c-Jun NH2-terminal kinase 1 (JNK1) expression attenuates (A) the inhibition of cell proliferation and (B) the promotion of apoptosis induced by sodium selenite (Se). The HL-60 cells were transfected with a control small interfering RNA (si-Control) or a small interfering RNA targeting human JNK1, and then treated with $250 \mathrm{~nm}$ Se for $48 \mathrm{~h}$. The cell proliferation inhibition rate was analyzed by MTS assay, and apoptosis was assayed by flow cytometry.

cells with $250 \mathrm{nM}$ Se for $48 \mathrm{~h}$. We found that the knockdown of JNK1 abrogated the inhibitory effects of Se on cell proliferation (Fig. 4A) and the induction of apoptosis (Fig. 4B).

Se induces changes in protein expression in HL-60 cells. To determine the potential mechanisms through which Se induces apoptosis and cell cycle arrest, we assayed the changes in the expression of the apoptosis-associated protein, JNK1, and the cell cycle regulators, p21, p27 and cyclin D1. Se (250 nM) significantly induced JNK1 phosphorylation even though the levels of total JNK1 were not altered (Fig. 5). In addition, the expression of p21 and p27 was increased (by approximately 100 - and 300-fold, respectively) and that of cyclin D1 was decreased by approximately 60-fold, compared to the untreated cells and those treated with $100 \mathrm{nM} \mathrm{Se}$.

\section{Discussion}

In this study, using an in vitro cell culture model, found that a higher concentration of Se inhibited HL-60 cell proliferation, induced HL-60 cell apoptosis and caused cell cycle arrest, and these effects of selenium were associated with enhanced JNK1 phosphorylation and increased p21/p27 expression (Fig. 6).

It has been reported that selenium exerts multiple anticancer effects, such as antioxidant (19,20), anti-inflammatory and/or 

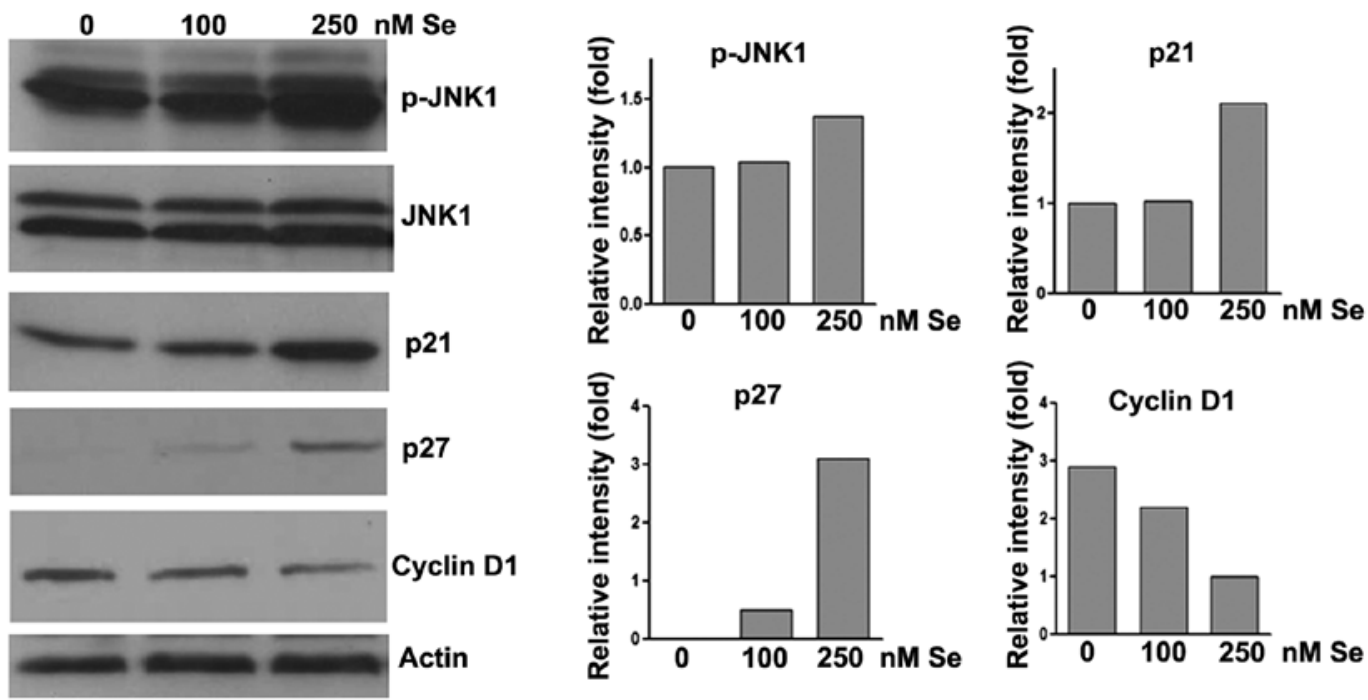

Figure 5. Sodium selenite (Se) increases the levels of phosphorylated JNK and the expression of p21 and p27, but deceases cyclin D1 expression in HL-60 cells. The HL-60 cells were treated with 0,100 or $250 \mathrm{nM}$ of for $48 \mathrm{~h}$ and collected for immunoblotting. The levels of phosphorylated JNK (p-JNK) were increased following treatment with $250 \mathrm{nM} \mathrm{Se}$, although the levels of total JNK were not altered. Bars on the right panel indicate the quantification of the immunoblotting intensities.

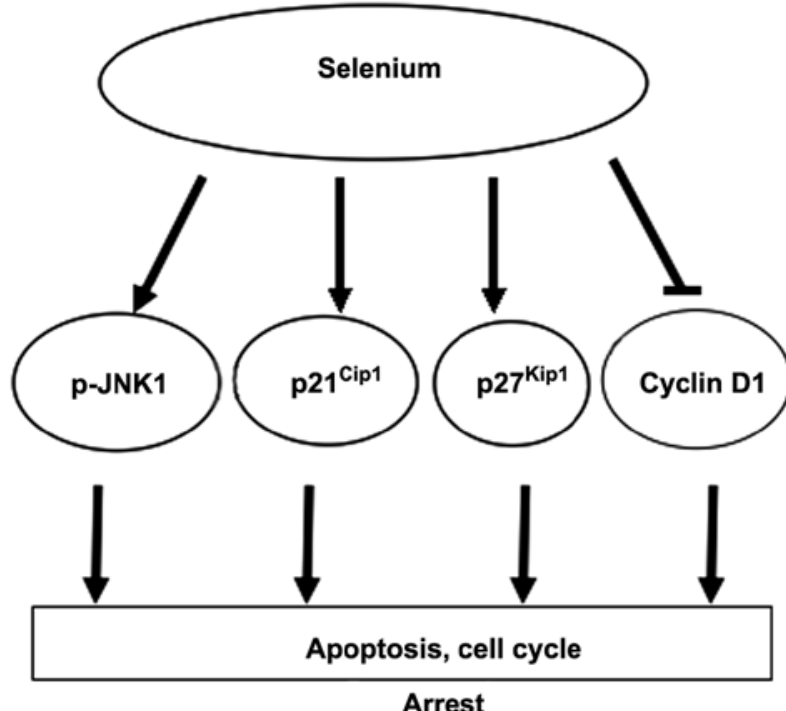

Figure 6. Novel anticancer mechanisms of sodium selenite in HL-60 cells. Se induces the phosphorylation of c-Jun NH2-terminal kinase 1 (JNK1), increases p21 and p27 expression, and deceases cyclin D1 expression, leading to HL-60 cell apoptosis and cell cycle arrest.

suppressive effects by inhibiting $\beta$-catenin through 1,4-phenylene bis (methylene) selenocyanate $(9,21,22)$, and increasing the phosphorylation of mitogen-activated protein kinase (MAPK) in prostate cancer cells through methylseleninic acid $(23,24)$. In our previous study, we demonstrated that tumor inhibition by $\mathrm{Se}$ in colorectal cancer was associated with the phosphorylation of JNK1 and the consequent inhibition of $\beta$-catenin and its transcriptional targets, c-myc, cyclin D1 and CDK4, leading to the induction of apoptosis and inhibition of cell proliferation. In addition, Cox 2 was almost completed eliminated by Se in mouse intestinal epithelial cells (10). In another recent study, using an Apc/p21 complex mouse model of colorectal cancer, we found that the combination of selenium and the non-steroidal antiinflammation drug, sulindac, led to the significant induction of the expression of p27 and p53 and JNK1 phosphorylation, as well as to the suppression of $\beta$-catenin and its downstream targets, also leading to a demythelation on the p21 promoter (25), an additional mechanism of selenium in cancer prevention, in which selenium showed a synergistic role with sulindac in exerting maximal inhibitory effects on tumor growth. This finding also provides an important chemopreventive strategy using a combination of anticancer agents, which has a great impact on cancer prevention and has a promising translational potential.

JNK1 plays important roles in the regulation of cell proliferation, differentiation and apoptosis in response to cellular stress and chemopreventive agents $(12,15,26,27)$. In the current study, we demonstrated that the selenium-induced inhibition of leukemia HL-60 cell proliferation and the induction of apoptosis was through JNK1 activation. Experiments using siRNA targerting JNK1 further demonstrated that the selenium-mediated effects on HL-60 cells required JNK1. Our data strongly suggest that JNK1 plays a critical role in the selenium-mediated chemoprevention of leukemia cells, in which JNK1 phosphorylation or activation may be one of the key factors through which selenium induces apoptosis.

Basic research and clinical trials have shown the strong tumor preventive effects of selenium (6), whereas the outcome of the Selenium and Vitamin E Cancer Prevention Trial (SELECT) showed that selenium or vitamin $\mathrm{E}$, alone or in combination did not prevent cancer but caused an increased risk of cancer and metabolism-associated diseases (28). This failure may be due to the different form of selenium used, as well as the dosage, and the baseline selenium status and genotypes (e.g., polymorphims) of selenium-containing proteins, such as glutathione peroxidase 1 (GPx1) and selenium-binding protein 1 (SBP1). Both proteins are selenium-contain proteins and are negatively regulated by each other, and this interaction plays very important roles in both cancer prevention and carcinogenesis (29).

The promotion of apoptosis and the induction of cell cycle arrest are major mechanisms of action of chemotherapeutic agents, in which cell cycle regulators, such as cyclins 
(e.g., cyclin D1 and cyclin E), cyclin-dependent kinases (e.g., cdk2, cdk4 and cdk6), and cyclin-dependent kinase inhibitors (e.g., p21, p27 and p16) (30) play crucial roles. In this study, we found that apart from the induction of apoptosis, selenium induced cell cycle arrest at the $\mathrm{S}$ phase. This effect was associated with the increased expression of p21 and p27 and the decreased expression of cyclin D1.

In conclusion, our data demonstrate that Se is effective in inhibiting HL-60 cell proliferation, promoting apoptosis and inducing cell cycle arrest in leukemia cells by targeting JNK1 and the cell cycle signaling pathway, which provides further evidence of the anticancer bioactivity of selenium and suggests that selenium may have additional usage beyond solid tumors. In addition, our data have improved our understanding of the mechanisms responsible for the selenium-induced anticancer effects and suggest a novel use of selenium in leukemia.

\section{Acknowledgements}

This study was supported by the National Natural Science Foundation of China (81272251), the Doctor Start-up Research Fund (100820 and 505011) and the Key Research Fund (ZD2011-18) from Xinxiang Medical University.

\section{References}

1. Siegel R, Ward E, Brawley O and Jemal A: Cancer statistics, 2011: the impact of eliminating socioeconomic and racial disparities on premature cancer deaths. CA Cancer J Clin 61: 212-236, 2011

2. Uğuz AC, Naziroğlu M, Espino J, Bejarano I, Gonzalez D, Rodriguez AB and Pariente JA: Selenium modulates oxidative stress-induced cell apoptosis in human myeloid HL-60 cells through regulation of calcium release and caspase- 3 and -9 activities. J Membr Biol 232: 15-23, 2009.

3. Ip C: Lessons from basic research in selenium and cancer prevention. J Nutr 128: 1845-1854, 1998.

4. Wang L, Bonorden MJ, Li GX, Lee HJ, Hu H, Zhang Y, Liao JD, Cleary MP and Lü J: Methyl-selenium compounds inhibit prostate carcinogenesis in the transgenic adenocarcinoma of mouse prostate model with survival benefit. Cancer Prev Res (Phila) 2: 484-495, 2009.

5. Facompre N and El-Bayoumy K: Potential stages for prostate cancer prevention with selenium: implications for cancer survivors. Cancer Res 69: 2699-2703, 2009.

6. Clark LC, Combs GF Jr, Turnbull BW, Slate EH, Chalker DK, Chow J, Davis LS, Glover RA, Graham GF, Gross EG, et al: Effects of selenium supplementation for cancer prevention in patients with carcinoma of the skin. A randomized controlled trial. Nutritional Prevention of Cancer Study Group. JAMA 276: 1957-1963, 1996.

7. Greenwald P, Anderson D, Nelson SA and Taylor PR: Clinical trials of vitamin and mineral supplements for cancer prevention. Am J Clin Nutr 85: 314S-317S, 2007.

8. Hawk ET and Levin B: Colorectal cancer prevention. J Clin Oncol 23: 378-391, 2005.

9. Peters U and Takata Y: Selenium and the prevention of prostate and colorectal cancer. Mol Nutr Food Res 52: 1261-1272, 2008.

10. Fang W, Han A, Bi X, Xiong B and Yang W: Tumor inhibition by sodium selenite is associated with activation of c-Jun NH2-terminal kinase 1 and suppression of beta-catenin signaling. Int J Cancer 127: 32-42, 2010.

11. Song Z, Tong C, Liang J, Dockendorff A, Huang C, Augenlicht LH and Yang W: JNK1 is required for sulindac-mediated inhibition of cell proliferation and induction of apoptosis in vitro and in vivo. Eur J Pharmacol 560: 95-100, 2007.
12. Tong C, Yin Z, Song Z, Dockendorff A, Huang C, Mariadason J, Flavell RA, Davis RJ, Augenlicht LH and Yang W: c-Jun NH2-terminal kinase 1 plays a critical role in intestinal homeostasis and tumor suppression. Am J Pathol 171: 297-303, 2007.

13. Davis RJ. Signal transduction by the JNK group of MAP kinases. Cell 103: 239-252, 2000.

14. Johnson GL and Lapadat R: Mitogen-activated protein kinase pathways mediated by ERK, JNK, and p38 protein kinases. Science 298: 1911-1912, 2002.

15. Hu D, Fang W, Han A, Gallagher L, Davis RJ, Xiong B and Yang W: c-Jun N-terminal kinase 1 interacts with and negatively regulates Wnt/beta-catenin signaling through GSK3beta pathway. Carcinogenesis 29: 2317-2324, 2008.

16. Yang WC, Mathew J, Velcich A, Edelmann W, Kucherlapati R, Lipkin M, Yang K and Augenlicht LH: Targeted inactivation of the $\mathrm{p} 21$ (WAF1/cip1) gene enhances Apc-initiated tumor formation and the tumor-promoting activity of a Western-style high-risk diet by altering cell maturation in the intestinal mucosal. Cancer Res 61: 565-569, 2001

17. van de Wetering M, Sancho E, Verweij C, de Lau W, Oving I, Hurlstone A, van der Horn K, Batlle E, Coudreuse D, Haramis AP, et al: The beta-catenin/TCF-4 complex imposes a crypt progenitor phenotype on colorectal cancer cells. Cell 111: 241-250, 2002.

18. Tetsu $\mathrm{O}$ and McCormick F: Beta-catenin regulates expression of cyclin D1 in colon carcinoma cells. Nature 398: 422-426, 1999.

19. Lü J and Jiang C: Selenium and cancer chemoprevention: hypotheses integrating the actions of selenoproteins and selenium metabolites in epithelial and non-epithelial target cells. Antioxid Redox Signal 7: 1715-1727, 2005.

20. Diwadkar-Navsariwala V andDiamond AM: The link between selenium and chemoprevention: a case for selenoproteins. J Nutr 134: 2899-2902, 2004.

21. Narayanan BA, Narayanan NK, Desai D, Pittman B and Reddy BS: Effects of a combination of docosahexaenoic acid and 1,4-phenylene bis(methylene) selenocyanate on cyclooxygenase 2 , inducible nitric oxide synthase and beta-catenin pathways in colon cancer cells. Carcinogenesis 25: 2443-2449, 2004.

22. Rao CV, Cooma I, Rodriguez JG, Simi B, El-Bayoumy K and Reddy BS: Chemoprevention of familial adenomatous polyposis development in the APC(min) mouse model by 1,4-phenylene bis(methylene) selenocyanate. Carcinogenesis 21: 617-621, 2000.

23. Hu H, Jiang C, Ip C, Rustum YM and Lü J: Methylseleninic acid potentiates apoptosis induced by chemotherapeutic drugs in androgen-independent prostate cancer cells. Clin Cancer Res 11: 2379-2388, 2005

24. Jiang C, Wang Z, Ganther H and Lu J: Distinct effects of methylseleninic acid versus selenite on apoptosis, cell cycle, and protein kinase pathways in DU145 human prostate cancer cells. Mol Cancer Ther 1: 1059-1066, 2002.

25. Bi X, Pohl N, Dong H and Yang W: Selenium and sulindac are synergistic to inhibit intestinal tumorigenesis in Apc/p21 mice. J Hematol Oncol 6: 8, 2013.

26. Hu D, Bi X, Fang W, Han A and Yang W: GSK3beta is involved in JNK2-mediated beta-catenin inhibition. PLoS One 4: e6640, 2009.

27. Bode AM and Dong Z: The functional contrariety of JNK. Mol Carcinog 46: 591-598, 2007.

28. Lippman SM, Klein EA, Goodman PJ, Lucia MS, Thompson IM, Ford LG, Parnes HL, Minasian LM, Gaziano JM, Hartline JA, et al: Effect of selenium and vitamin $\mathrm{E}$ on risk of prostate cancer and other cancers: the Selenium and Vitamin E Cancer Prevention Trial (SELECT). JAMA 301: 39-51, 2009.

29. Fang W, Goldberg ML, Pohl NM, Bi X, Tong C, Xiong B, Koh TJ, Diamond AM and Yang W: Functional and physical interaction between the selenium-binding protein 1 (SBP1) and the glutathione peroxidase 1 selenoprotein. Carcinogenesis 31: 1360-1366, 2010.

30. Chu IM, Hengst L and Slingerland JM: The Cdk inhibitor p27 in human cancer: prognostic potential and relevance to anticancer therapy. Nat Rev Cancer 8: 253-267, 2008. 